\title{
Analysis of Intraarticular Application of Mesenchymal Stem Cells and Platelet Rich Plasma in Treatment for Knee Osteoarthritis
}

\section{Julieth Kristel Olague Franco* and Ismael Moreno Castillo}

Department of Orthopedics and Traumatology, ISSSTE Mexico, ISSSTE, Instituto de Seguridad y Servicios Sociales de los Trabajadores del Estado, Mexico

*Corresponding Author: Julieth Kristel Olague Franco, Department of Orthopedics and Traumatology, Instituto de Seguridad y Servicios Sociales de los Trabajadores del Estado, Mexico.

Received: July 25, 2019; Published: August 13, 2019

\begin{abstract}
The application of human mesenchymal cells (MSC) and platelet-rich plasma (PRP) in the knee represents a less invasive therapy with a promising effectiveness. The purpose of this study is to identify the efficacy and safety in the treatment of osteoarthritis with intra-articular application of MSC compared to PRP and a placebo group. 50 patients with osteoarthritis of the knee were selected, the patients were divided into 5 groups with 10 members each; three of these groups were from MSC: the low-dose $\left(1.0 \times 10^{7}\right.$ cells), mid-dose $\left(5.0 \times 10^{7}\right)$, and high-dose $\left(1.0 \times 10^{8}\right)$; another group for PRP and the last for placebo group, and were infiltrated a single occasion. All patients were followed up with Western Ontario and McMaster Universities Osteoarthritis index (WOMAC) and Lequesne index at the beginning of the study and at the 3, 6, 9 and 12 months as primary controls, As well as arthroscopies and radiographs were randomly performed at the beginning and at the end of the study as secondary controls. There was no treatment related adverse event. The results of the primary controls showed a significant improvement for the low-dose and mid-dose of MSC groups, with a peak in improvement at 9 months. Arthroscopy showed that the size of cartilage defect decreased in the medial femoral and medial tibial condyles of the mid-dose group. These results showed that the application of mid-dose of MSC in osteoarthritis improves the function and pain in the knee, compared to the placebo group and other types of treatment as the PRP. We conducted a proof-of-concept phase I/II clinical trial.
\end{abstract}

Keywords: Osteoarthritis; Platelet Rich Plasma; Mesenchymal Stem Cells

\section{Introduction}

Osteoarthritis (OA) of the knee is a common chronic joint disease affecting nearly $10 \%$ of the population [1]. This condition ranks fifth among the top causes of years lost to disability in highincome countries and ninth in low- and middle-income countries [2]. In men ages more than 50 years, osteoarthritis represents the second leading cause of work disability. Furthermore, osteoarthritis is responsible for approximately $2 \%$ of all public health expenses [3] and large indirect costs derived from productivity decreases [4]. Its prevalence increases with ageing as well as among obese persons; therefore, the burden due to OA will increase substantially in the near future because of the demographic changes and obesity epidemic [5-7]. Many treatments have been proposed but resulted in poor clinical results without cartilage repair [8]. Articular replacement with prostheses is only recommended as the last treatment option. The American Academy of Orthopaedic Surgeons recommends only physical and educational therapy, symptomatic treatment with acetaminophen or nonsteroidal anti-inflammatory drugs, and sometimes local corticosteroid injection [9].

The mesenchymal stem cells (MSC) are located primarily in the bone marrow and have the ability to give rise to various cell types such as fibroblasts or medullary reticular cells, adipocytes, osteoblasts and chondrocytes $[10,11]$. Studies conducted both in vivo and in vitro have demonstrated the plasticity of these cells, giving rise to non-hematopoietic cells such as myocytes, tenocyte and chondral cells, the latter being an option for the treatment of knee osteoarthritis because the pathogenesis of this problem is the chondral destruction in the natural history of this disorders $[12,13]$.

The platelet-rich plasma (PRP) has been configured as a perspective for improving clinical and structural outcomes by delivering a high concentration of growth factors that mediate cartilage 
healing and remodeling, PRP is a plasma fraction obtained by apheresis procedures and centrifugation, characterized by a high platelet concentration and growth factors, polypeptide substances that play an important role in regulating the growth, differentiation and phenotype of multiple cell types, as well as actively participates in the processes of repair and cicatrization [14]. This is the most important activity expected, tissue repair at a chondral level specifically for the articular cartilage of the knee [14].

\section{Materials and Methods}

Study design and patients

We conducted a proof-of-concept phase I/II clinical trial to assess the safety and the efficacy of intra-articular injection of MSC, PRP and solution saline like placebo in patients with knee osteoarthritis, accomplished between March 2015 and May 2016 in the Regional Hospital Valentin Gomez Farias, Jalisco, Mexico. The protocol was approved by the ethics committee of our hospital. All participants provided written institutional informed consent.

50 patients were selected with chronic knee osteoarthritis unresponsive to conventional treatments. The inclusion criteria were: patient age, with idiopathic osteoarthritis of the knee of grade 2 or more according to Kellgren-Lawrence criteria, physical activity (Lequesne Index= Moderate), the Body Mass Index (BMI= $<35$ ) and Western Ontario and McMaster University Osteoarthritis Index (WOMAC Index= >40). In exclusion criteria were taken into account patients with rheumatoid arthritis, ligamentous instability and antecedents of cancer. The study involved three phases;

\section{Phase 1}

The first included the recruitment of patients the final check of compliance with inclusion criteria (anteroposterior and lateral radiographs of knee and body mass index volume); was applied the WOMAC index consisting of a measure to assess pain, stiffness and functionality. A result greater than 40 would be significant for a functional limitation caused by pain. The assessment of physical activity in this study is based on the Lequesne index, which was designed to assess the functional capacity and pain of patients with osteoarthritis of the hip and knee. Questions are asked to determine the degree of pain, the distance that can be traveled by the patient and how it affects the activities of daily life of the patient. A result greater than 40 is associated with the limitation of physical activity in daily life.

$15 \mathrm{ml}$ of whole blood is extracted from all patients, to avoid that the patients knew the group to which they were destined.

Four patients were randomly selected from the fifty patients in the study, and the arthroscopic examination of the knee was performed; Lesions of the articular cartilage were measured with a calibrated arthroscopic probe and classified according to the international cartilage repair society (ICRS) cartilage injury classification [18]. No debridement, synovectomy or meniscectomy were performed during arthroscopy, and no drainage was used. A single orthopedic surgeon performed all procedures. Postoperative rehabilitation consisted on passive exercises and walk for less than 20 minutes from the seventh day of the procedure.

\section{Phase 2}

From the 50 patients were performed 5 groups with 10 patients each group (MSC $1.0 \times 10^{7}$, MSC $5.0 \times 10^{7}$, MSC $1.0 \times 10^{8}$, Platelet Rich Plasma and placebo group) (Table 1).

\begin{tabular}{|c|c|}
\hline \multicolumn{2}{|r|}{ Patients Group } \\
\hline Group 1 & Mesenchymal stem cells $1.0 \times 10^{7}$ \\
\hline Group 2 & Mesenchymal stem cells $5.0 \times 10^{7}$ \\
\hline Group 3 & Mesenchymal stem cells $1.0 \times 10^{8}$ \\
\hline Group 4 & Platelet-Rich Plasma \\
\hline Group 5 & Placebo \\
\hline
\end{tabular}

Table 1: Patients Group.

This intra-articular application was performed by medial parapatellar, the intervention was carried out with the standards of aseptic and antiseptic, the placement was carried out in the operating room for the safety of the sample.

The only thing that was solicited to the patients was moderate rest for 2 days.

For the four patients who underwent arthroscopy, they were randomly assigned to the MSC and PRP groups, only except for the placebo group.

Phase 3

At this consists in a control consultation for the five groups, this consists in carrying out of new account rates of Lequesne and WOMAC index, to analyze the progress or changes in physical activities and whether or not symptomatic improvement, these controls were performed on all patients every 3, 6, 9 and 12 months after the application.

For the four patients who underwent arthroscopy, a new arthroscopy was performed at 12 months after implantation, the articular cartilage were measured with a calibrated arthroscopic probe and classified according to ICRS; no debridement, synovectomy or meniscectomy were performed during arthroscopy, and no drainage was used. 
Mesenchymal stem cells

Cell isolation and expansion were performed in the LCELLS LAB Cell Production Unit with approval of the Comisión Federal para la Protección contra Riesgos Sanitarios (12-TR-14-039-001). The samples were transported to the Cell Production Unit at $4^{\circ} \mathrm{C}$ to $12^{\circ} \mathrm{C}$ within 12 hours of harvesting. The mononuclear cell fraction was isolated by density-gradient centrifugation, resuspended, and cultured in MSC expansion culture medium in 175-cm2 tissue culture flasks, with periodic washing to remove nonadherent cells. At the end of this period (21-24 days), cells were harvested, resuspended in Ringer's lactate solution containing 0.5\% human albumin (CSL Behring GmbH, Marburg, Germany) and $5 \mathrm{mM}$ glucose. The antigenic profile conformed to the International Society for Cellular Therapy criteria for MSCs their preparation and manipulation of these cells were regulated by strict health standards of our national health system [16].

\section{Platelet rich plasma}

For the preparation of platelet rich plasma, $20 \mathrm{ml}$ of whole blood is removed from the all patients and centrifuged blood is received in sterile tubes with $3.8 \%$ sodium citrate as an anticoagulant. Centrifuge at 1,800 rpm for $10 \mathrm{~min}$ with varying concentrations of platelet-rich plasma. Said plasma is centrifuged again for 8 minutes at $1800 \mathrm{rpm}$ at room temperature. The result is approximately $4 \mathrm{ml}$ of platelet-rich plasma. Subsequently, plasma fractions obtained are separated by pipetting very careful not to create turbulence [17].

\section{Results}

The 50 selected patients had been unresponsive to conservative treatment (physical and medical) for at least 12 months. No serious adverse events occurred. Only six patients in the PRP group present edema and hyperemia the first three days after inoculation (these were treated with physical means) No patient left the study due to adverse events. There were no clinically important trends in the results of physical examination, vital signs, laboratory test during the study.

\section{Clinical outcomes}

All patients in the present study had a marked improvement; even the placebo group showed a slight improvement at 3 months, as the other groups had an improvement above the placebo group with a peak of improvement at 6 months for PRP and 9 months for MSC.

Never the less the PRP and MSC (high-dose $1.0 \times 10^{8}$ ) group was associated with not significant improvement on WOMAC and Lequesne index compared with initial evaluation and placebo group.

The results from the WOMAC index (Figure 1) from the initial evaluation over 12 months was $0.84 \%$ to improve from $59.5 \pm 5.2$ to $59 \pm 6.1(\mathrm{p}=0.005)$ to Placebo group; $56.96 \%$ in the PRP group from $79 \pm 5.7$ to $34 \pm 6.4$ (p=0.001); $36.44 \%$ in MSC Low-dose 1.0 $\mathrm{x} 107$ from $57.9 \pm 3.1$ to $36.8 \pm 3.7$ ( $\mathrm{p}<.001$ ); $79.93 \%$ in MSC Middose $5.0 \times 107$ from $59.8 \pm 4.1$ to $12 \pm 4.3$ (p<.001) and $14.71 \%$ in MSC high-dose $1.0 \times 108$ from $59.8 \pm 6.8$ to $51 \pm 7(p=.020)$. With a peak of improvement for the placebo group and PRP at six months, and a regression of the improvement later; the peak improvement in the MSC group was observed at 9 months to maintain such improvement, except for the high dose which had a regression of the improvement at 12 months.

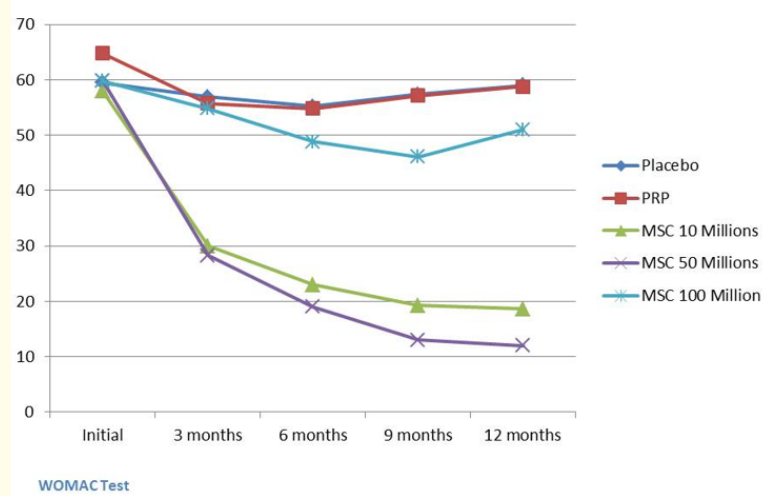

Figure 1: Results of WOMAC Test.

The results from the Lequesne index (Figure 2) from the baseline over 12 months was $2.87 \%$ to improve from $15.65 \pm 1.3$ to $15.2 \pm 1.7(\mathrm{p}=.025)$ to Placebo group; $61.14 \%$ in the PRP group from $17.5 \pm 1.4$ to $6.8 \pm 1.4$ ( $\mathrm{p}=.005$ ); $33.2 \%$ in MSC Low-dose $1.0 \mathrm{x}$ 107 from $12.65 \pm 0.7$ to $8.45 \pm 0.9$ (p .001), $70.70 \%$ MSC Mid-dose $5.0 \times 107$ from $14.85 \pm 0.4$ to $4.35 \pm 0.7(\mathrm{p}<.001)$, and $11.82 \%$ in MSC high-dose $1.0 \times 108$ from $15.65 \pm 1.7$ to $13.8 \pm 1.3$ (p =.01). A peak of improvement was observed for the placebo group at six months and three months for PRP group, to subsequently have a decrease in the improvement; the peak of improvement in the MSC group was observed at 9 months to maintain such improvement.

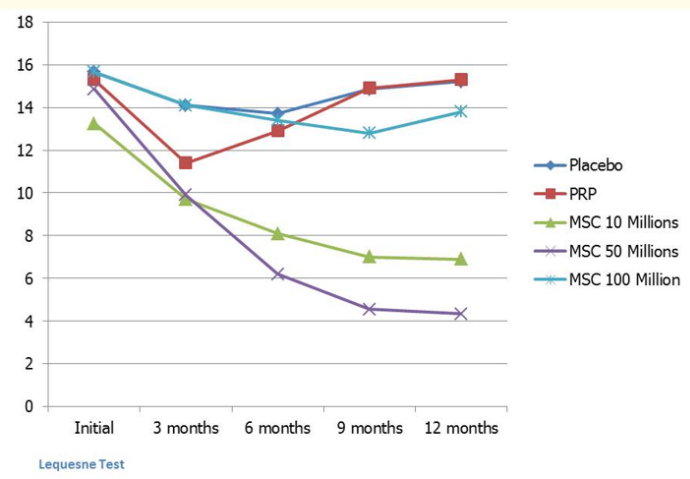

Figure 2: Results of Lequesne Test. 


\section{Radiological outcomes}

Patients in each group had similar Kellgren-Lawrence grade, joint space width, mechanical axis, and anatomical axis did not change significantly over 12 months in all dose groups (not illustrated).

\section{Arthroscopy}

For the final evaluation of the articular cartilage, an arthroscopy was performed 12 months after the infiltration of PRP or MSC, to assess the quality of the cartilage and to perform measurements according to ICRS grade. To the four patients who underwent arthroscopy prior to the inoculation of PRP and MSC, a second control arthroscopy was performed 12 months after inoculation, following the same previous parameters.

Macroscopic improvement in the articular cartilage was observed in the three patients who received MSC (Figure 3); however, in the patient who was inoculated PRP, no improvement or changes in cartilage measurements were observed (not illustrated). The improvement shown in patients with MSC application was demonstrated macroscopically in the regenerated cartilage, in the more severely degenerate areas with ICRS grade 3 in the femoral and tibial condyles of the medial compartment, a slight improvement was observed in less degenerate areas such as the lateral compartment and the patella. Regenerated cartilage looked glossy white with a smooth surface. Cartilage felt firm like healthy articular cartilage in the medial femoral condyle, whereas it was less firm in the medial tibial condyle. No loose body, hypertrophy, or abnormal calcification was identified. The size of cartilage defect in the lateral femoral and tibial condyle and the patella did not change in all dose groups over one year.

The cartilage defect measured (calibrated probe) demonstrated a reduction of the cartilage defect from $987.4 \pm 134.6 \mathrm{~mm}^{2}$ to $743.1 \pm 125.9 \mathrm{~mm}^{2}$ in the medial compartment $(25 \%$ decrease; $\mathrm{p}=$ .06) for MSC Low-dose $1.0 \times 107$; in the Mid-dose 5.0 x 107 group the cartilage defect from $1145.7 \pm 47.3 \mathrm{~mm}^{2}$ to $522.4 \pm 41.6 \mathrm{~mm} 2$ (54\% decrease; $\mathrm{p}=.03$ ) and in the MSC high-dose $1.0 \times 10^{8}$ group the cartilage defect from $1207.4 \pm 83.2 \mathrm{~mm}^{2}$ to $1100.2 \pm 55.3 \mathrm{~mm}^{2}$ ( $9 \%$ decrease; $p=.09$ ). The size of cartilage defect in the lateral femoral and tibial condyle and the patella did not change in all doses groups (Figure 4).

\section{Statistical analysis}

The significance of differences was assessed either by Student's $t$ test or by one-way analysis of variance (ANOVA) and the corresponding nonparametric tests. The analysis was performed using SAS version 9.2 (SAS Institute, Inc., Cary, NC).

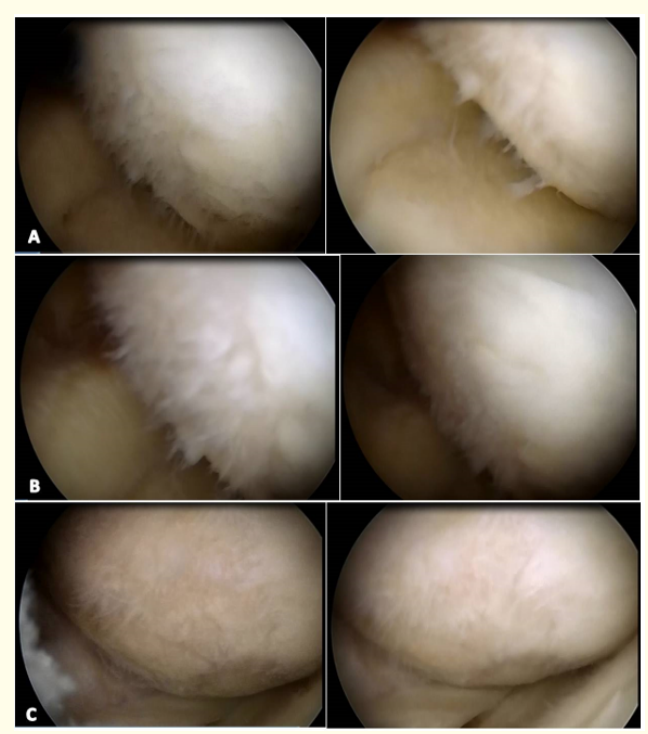

Figure 3: Arthroscopic control, The images show the medial compartment of the knee and in the images from the left shown the initial arthroscopy and right is displayed arthroscopy performed 12 months after the infiltration; in the image (A) the patient pertaining to the group of MSC $1.0 \times 10^{7}$; in the image (B) the patient pertaining to the group of MSC $5.0 \times 10^{7}$; in the image (C) the patient pertaining to the group of MSC $1.0 \times 10^{8}$.

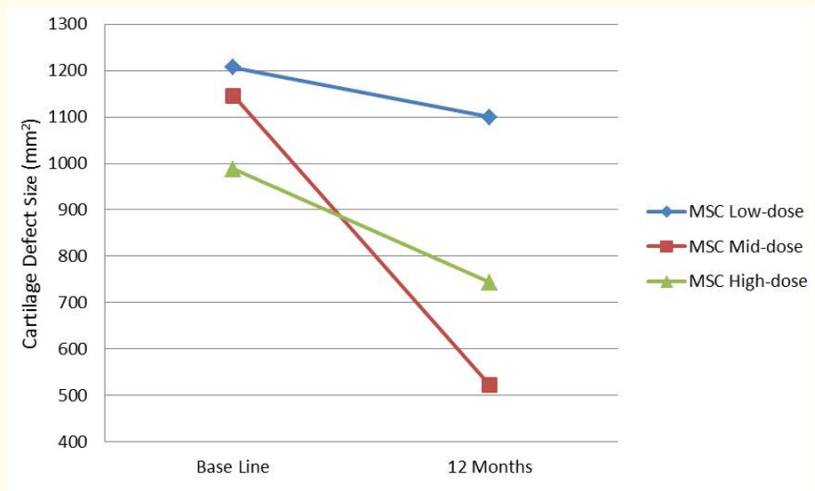

Figure 4: Cartilage Defect Size.

\section{Discussion}

The beneficial effects of MSC treatment continue to be studied. Chondrocytes induce differentiation of cultured MSCs toward a chondrocyte phenotype [19]. Proliferation and differentiation of MSCs to chondrocytes also happen with MSCs injected into knee joints [20], stimulate cultured cells to proliferate and synthesize extracellular matrix [21-23] and promote expression of collagen II and other chondrogenic genes by host cells [24]. Furthermore, 
MSCs have a well-known immunomodulatory effect $[25,26]$ and can induce anti-inflammatory cytokine production [27]. These data indicate that MSCs may help analgesia by reducing inflammation. Because the analgesic effect is more evident than anatomic restoration, we conclude that the trophic and anti-inflammatory effects of MSCs on the damaged tissue may occur more quickly than the regenerative effects. When PRP is injected into the injured site, platelets are activated by endogenous thrombin and/or intraarticular collagen [28]. The mediators that are secreted by PRP inoculation act as anti-catabolic and anti-inflammatory agents [28, 29]. Moreover, favor the stabilization of cartilage by controlling the metabolic functions of chondrocytes and subchondral bone, maintaining the homeostasis between the synthesis and degradation of proteoglycans, and stimulating the proliferation of chondrocytes $[30,31]$. It was also found that platelet growth factors stimulate synovial fibroblasts to synthesize hyaluronic acid [30]. Taking into account all the qualities that these therapies have, this study was conducted to analyze and evaluate the effectiveness and safety in the application of these two types of therapies for osteoarthritis in the knee, which have the characteristic of being a non-surgical treatment.

As seen in different studies [33], the effectiveness of these therapies are promising, and with the results of this study, we agree that they are a treatment that does not present adverse effects and that improve the symptomatology in osteoarthritis of knee, but must mention the following:

No significant improvement was observed in patients who infiltrated PRP, even had results very similar to the placebo group, so it is suggested to further study its effectiveness in osteoarthritis of the knee.

Patients with MSC infiltration should make two special mentions, first, symptomatic and functional improvement was observed in all three groups, but not with the results expected or mentioned in other studies [33,34], where it was expected a greater Effectiveness in patients with high dose of MSC was only slightly above the PRP and placebo groups, where a better outcome was observed in the medium dose group. The second mention is for the growth of articular cartilage, which was present in the three groups, having a higher cell growth in the med dose group; other studies are needed to analyze the cell type present in the regenerated cartilage.

\section{Conclusion}

No improvement was seen with intra-articular PRP injections in the treatment of $\mathrm{OA}$ of the knee in terms of pain relief and improvement of function analyzed at 3, 6 and 12 months of follow- up, and their results were very similar compared to placebo saline injection.

Intra-articular MSC injections mainly the Mid-dose $5.0 \times 107$ group probably are more efficacious in the treatment of knee $\mathrm{OA}$ in terms of pain relief and improvement of function without causing adverse events analyzed at 3, 6 and 12 months of follow-up, compared with other injections, including saline placebo and PRP. Radiological and arthroscopic measures consistently demonstrated regeneration of hyaline-like articular cartilage. These results are promising to have a new treatment in knee $\mathrm{OA}$ and also to continue performing randomized clinical studies and find the optimum dose of MSC.

\section{Bibliography}

1. Helmick CG., et al. "Estimates of the prevalence of arthritis and other rheumatic conditions in the United States. Part I". Arthritis and Rheumatology 58 (2008): 15-25.

2. Wittenauer R., et al. "Update on 2004 Background Paper, BP 6.12 Osteoarthritis". Geneva: World Health Organization (2013)

3. Le Pen C., et al. "Financial cost of osteoarthritis in France. The "COART" France study". Joint Bone Spine 72 (2005): 567.

4. Hermans J., et al. "Productivity costs and medical costs among working patients with knee osteoarthritis". Arthritis Care Research (Hoboken) 64 (2012): 853.

5. Arden N and Nevitt MC. "Osteoarthritis: epidemiology". Best Practice and Research: Clinical Rheumatology 20 (2006): 3-25.

6. Blagojevic M., et al. "Risk factors for onset of osteoarthritis of the knee in older adults: a systematic review and metaanalysis". Osteoarthritis Cartilage 18 (2010): 24-33.

7. Grotle M., et al. "Obesity and osteoarthritis in knee, hip and/ or hand: an epidemiological study in the general population with 10 years follow-up". BMC Musculoskeletal Disorders 9 (2008): 132.

8. Hawker GA., et al. "Osteoarthritis year 2010 in review: nonpharmacologic therapy". Osteoarthritis Cartilage 19 (2011): 366.

9. American Academy of Orthopaedic Surgery. "Treatment of Osteoarthritis of the Knee (Non-arthroplasty)". Full Guideline. Rosemont, IL: American Academy of Orthopaedic Surgeons (2008).

10. Wakitani S., et al. "Human autologous culture expanded bone marrow mesenchymal cell transplantation for repair of cartilage defects in osteoarthritic knees". Osteoarthritis Cartilage 10 (2002): 199-206. 
11. Kuroda R., et al. "Treatment of a full-thickness articular cartilage defect in the femoral condyle of an athlete with autologous bone-marrow stromal cells". Osteoarthritis Cartilage 15 (2007): 226-231.

12. Mokbel AN., et al. "Homing and reparative effect of intraarticular injection of autologous mesenchymal stem cells in osteoarthritic animal model". BMC Musculoskeletal Disorders 12 (2011): 259.

13. Emadedin M., et al. "Intra-articular injection of autologous mesenchymal stem cells in six patients with knee osteoarthritis". Archives of Iran Medicine 15 (2012): 422-428.

14. U Noth., et al. "Technology insight: adult mesenchymal stem cells for osteoarthritis therapy". Nature Reviews Rheumatology 47 (2008): 371-380.

15. F Barry and M Murphy. "Mesenchymal stem cells in joint disease and repair". Nature Reviews Rheumatology 910 (2013): 584-594.

16. Dominici M., et al. "Minimal criteria for defining multipotent mesenchymal stromal cells. The International Society for Cellular Therapy position statement". Cytotherapy 8 (2006): 315.

17. Wang-Saegusa A., et al. "Infiltration Of Plasma Rich In Growth Factors For Osteoarthritis Of The Knee Short-Term Effects On Function And Quality Of Life". Archives of Orthopaedic and Trauma Surgery 131 (2010): 311-317.

18. Brittberg M and Peterson L. "Introduction of an articular cartilage classification”. ICRS Newsletter 1 (1998): 5-8.

19. Hwang NS., et al. "Chondrogenic priming adipose-mesenchymal stem cells for cartilage tissue regeneration". Pharma Research 28 (2011): 1395.

20. Sato M., et al. "Direct transplantation of mesenchymal stem cells into the knee joints of Hartley strain guinea pigs with spontaneous osteoarthritis". Arthritis Research Therapy 14 (2012): R31.

21. Acharya C., et al. "Enhanced chondrocyte proliferation and mesenchymal stromal cells chondrogenesis in coculture pellets mediate improved cartilage formation". Journal of Cell Physiology 227 (2012): 88.

22. Wu L., et al. "Trophic effects of mesenchymal stem cells in chondrocyte co-cultures are independent of culture conditions and cell sources". Tissue Engineering Part A 18 (2012): 1542 .

23. Horie M., et al. "Intra-articular injection of human mesenchymal stem cells (MSCs) promote rat meniscal regeneration by being activated to express Indian hedgehog that enhances expression of type II collagen". Osteoarthritis Cartilage 20 (2012): 1197
24. Qing C., et al. "Co-culture of chondrocytes and bone marrow mesenchymal stem cells in vitro enhances the expression of cartilaginous extracellular matrix components". Brazilian Journal of Medical and Biological Research 44 (2011): 303.

25. Le Blanc K and Ringden O. "Immunomodulation by mesenchymal stem cells and clinical experience". Journal of International Medicine 262 (2007): 509.

26. Aggarwal S and Pittenger MF. "Human mesenchymal stem cells modulate allogeneic immune cell responses". Blood 105 (2005): 1815.

27. Yang SH., et al. "In vitro study on interaction between human nucleus pulposus cells and mesenchymal stem cells through paracrine stimulation". Spine 33 (2008): 1951.

28. Harrison S., et al. "Platelet activation by collagen provides sustained release of anabolic cytokines". American Journal of Sports Medicine.

29. Van Buul GM., et al. "Platelet-rich plasma releasate inhibits inflammatory processes in osteoarthritic chondrocytes". American Journal of Sports Medicine 39 (2011): 2362-2370.

30. Anitua E., et al. "Platelet-released growth factors enhance the secretion of hyaluronic acid and induce hepatocyte growth factor production by synovial fibroblasts from arthritic patients". Rheumatology 46 (2007): 1769-1772.

31. Schmidt MB., et al. "A review of the effects of insulin-like growth factor and platelet-derived growth factor on in vivo cartilage healing and repair". Osteoarthritis Cartilage 14 (2005): 403-12.

32. Nejadnik H., et al. "Autologous bone marrow-derived mesenchymal stem cells versus autologous chondrocyte implantation: An observational cohort study". American Journal of Sports Medicine 38 (2010): 1110-1116.

33. Kuroda R., et al. "Treatment of a full-thickness articular cartilage defect in the femoral condyle of an athlete with autologous bone-marrow stromal cells". Osteoarthritis Cartilage 15 (2007): 226-231.

34. Shen L., et al. "The temporal effect of platelet-rich plasma on pain and physical function in the treatment of knee osteoarthritis: systematic review and meta-analysis of randomized controlled trials". Journal of Orthopaedic Surgery and Research 2312.1 (2017): 16.

\section{Volume 2 Issue 9 September 2019 (C) All rights are reserved by Julieth Kristel Olague Franco and Ismael Moreno Castillo.}

